Ursache beruht es nun auch, dass Isomerieen baufig verschwinden, sobald kohlenstoff haltige Gruppen durch unorganische Radicale oder durch Metalle ersetzt werden. Schwerlich wird es jemals gelingen, dem Cyanmetall ein Isocyanmetall, der Cyansăure eine Isocyansăure an dłe Seite zu stellen, oder die Isomerie von Rhodanåthern und Senfölen, von Salpetrigsäureäther und Nitroäthan auf zugehörige Metallsalze auszudehnen. Und so ist es im Grunde gleichgūltig, ob man 2. B. die Cyanmetalle als $M-\cdots C N$ oder als $M$---NC betrachtet, wăhrend man doch Alkylcyanide und -Isocyanide neben einander aus ibnen gewinnt.

608. W. Roser: Beobachtungen in der Campherreihe. I.

[Aus dem chemischen Institut zu Marburg.]

(Eingegangen am 26. November.)

Bildung der Camphansăure.

Als nach der Vorschrift von Wreden Camphersăure aus Campher durch Oxydation mit Salpetersäure dargestellt und ihre Mutterlauge, nach Verdampfen der Salpetersäure, zur Entfernung der Camphoronsăure mit Barythydrat neutralisirt worden war, beobachtete ich beim Concentriren derselben die Ausscheidung eines schön krystallisirenden Baryumsalzes, welches bei der Analyse folgende Zahlen lieferte:

$0.3016 \mathrm{~g}$ gaben bei $100^{\circ} 0.0350$ Wasser und $0.1168 \mathrm{BaSO}_{4} 0.3766 \mathrm{~g}$, gaben 0.0441 Wasser.

\begin{tabular}{cllc}
\multicolumn{2}{c}{ Berechnet } & \multicolumn{2}{c}{ Gefunden } \\
für $\left(\mathrm{C}_{10} \mathrm{~B}_{13} \mathrm{O}_{4}\right)_{2} \mathrm{Ba}+4 \mathrm{H}_{2} \mathrm{O}$ & \multicolumn{2}{c}{$11.71 \mathrm{pCt}}$. \\
$\mathrm{H}_{2} \mathrm{O}$ & 11.94 & 11.6 & 1.6 \\
$\mathrm{Ba}$ & 22.72 & 22.77 & -
\end{tabular}

Aus diesem Baryumsalz wurde eine gegen $200^{\circ}$ schmelzende, salmiakß̌hnlich krystallisirende, an der Luft verwitternde Säure gewonnen, deren directer Vergleich mit der aus Bromcamphersäureanhydrid dargestellten $\mathrm{Camphansäure}^{1}$ ) sie als mit dieser identisch erkennen liess.

1) Das Baryumsalz der Camphersāure wurde vor Kurzem von Woringe $r$ (Ann. Chem. Pharm. 227.5) beschrieben, nach ihm enthălt es $3 \frac{1 / g}{2}$ Molekũal Wasser, er fand 10.5 und $11.3 \mathrm{pCt}$. Wasser. 


\section{Camphocarbonsă are (Campherkohlensãure).}

Durch die Einwirkung von Natrium und Kohlensăure auf Champher erhielt Ba abign y ${ }^{1}$ ) neben Borneolkohlensaure die Camphocarbonsä ure, welcher nach ihrer Entstehungsweise die Formel $\mathrm{C}_{10} \mathrm{H}_{15} \mathrm{O} . \mathrm{COOH}$ zugeschriehen werden musste. Ueber die nähere Constitution der Săure hat man sich nicht geänssert, die Frage nach derselben fallt zusammen mit derjenigen nach der Constitution des Camphers, wenn man denselben als Keton auffasste, musste jene Săure wohl als Ketonsăure betrachtet werden und ihr Verhalten, nămlich der leicht stattfindende Zerfall in Campher und Kohlensäure liess vermuthen, dass sie eine $\beta$-Ketonsäure wie die Acetessigsäure sei. Die Beobachtungen Silva's ${ }^{2}$ ) und von R. Schiff und J. Puliti ${ }^{3}$ ) betreffend die Bromund Chlorcamphocarbonsãure wiedersprechen dieser Auffassung nicht; J. K a c hler und Spitzer ${ }^{4}$ ), welche die Camphocarbonsäure (Campherkohlensăure) ausführlich untersuchten und auch ihre Bildungsweise aus Bibromcampher ${ }^{5}$ ) durch Einwirkung von Natrium und Kohlensiure kennen lehrten, schliessen dagegen aus der Existenz eines Natriumsalzes von der Zusammensetzung $\mathrm{C}_{22} \mathrm{H}_{31} \mathrm{NaO}_{6}$, dass die Molekulargrösse jener Säure zu verdoppeln sei und dass sie kein Carboxyl enthalte, weil bei der Einwirkung ron Phosphorpentachlorid auf dieselbe ein sauerstofffreies Chlorid $\mathrm{C}_{22} \mathrm{H}_{28} \mathrm{Cl}_{8}$ entsteht.

Diese Folgerung schien mir indessen nicht genügend begründet und ich beabsichtigte Versuche, in die Camphocarbonsäure Alkyle einzuführen wie in den Acetessigäther; dies ist mir zwar nicht gelungen, Bildungsweise und Eigenschaften des Aethers der Camphocarbonsilure dürften jedoch beweisen, dass derselben die früher angenommene Molekülgrösse $\mathrm{C}_{11} \mathrm{H}_{16} \mathrm{O}_{3}$ zukommt und dass sie auch ein Carboxyl enthält.

Den Aether der Camphocarbonsäure erbălt man leicht auf gewöhnliche Weise durch Einleiten von Chlorwasserstoff in die alkoholische Lősung der Saure; nach dem Abdestilliren des Alkohols durch Wasser abgeschieden mit Natriumcarbonatlösung und Wasser gewaschen und sodann mit Chlorcalcium getrocknet destillirt der Aether unter nur geringfügiger Zersetzung (Geruch nach Campher) bei $276^{\circ}$ (uncorr.) als farblose Flüssigkeit, deren specifisches Gewicht bei $15^{\circ}$ zu 1.052 bestimmt wurde.

$0.2499 \mathrm{~g}$ gaben 0.6388 Kohlensăure und 0.2046 Wasser.

1) B a u bigny, Zeitschr. f. Chem. 1868, IV., 482.

2) de Santos e Silva, diese Berichto VI, 1092.

3) R. Schiff u. S. Puliti, diese Berichte XVI, 887.

4) Kachler and Spitzer, Wiener Monatshefte 1881, II., 233.

5) Dasselbe 1882, 1II., 212. 


$\begin{array}{lcc}\text { Ber. für } \mathrm{C}_{13} \mathrm{H}_{20} \mathrm{O}_{3} & \text { Gefunden } \\ \text { C } & 69.64 & 69.96 \text { pCt. } \\ \text { H } & 8.92 & 9.09\end{array}$

Der Siedepunkt des Aetbers $276^{\circ}$ schliesst wohl die Möglichkeit aus, dass in demselben eine Verbindung der Zusammensetzung $\mathrm{C}_{26} \mathrm{H}_{40} \mathrm{O}_{6}$ vorliege; der Versuch eine Dumpfdichtebestimmung zur Feststellung des Molekulargewichts zu machen, schien mir überflüssig zu sein. Die Existenz des von Kachler und Spitzer beobachteten sauren Salzes $\mathrm{C}_{11} \mathrm{H}_{15} \mathrm{NaO}_{3} \cdot \mathrm{C}_{11} \mathrm{H}_{16} \mathrm{O}_{3} \mathrm{kann}$ auch in diesem Fall nicht maassgebend sein für die Beurtheílung der Molekulargrösse der Camphocarbonsäure.

Lässt man den Camphocarbonsäureäther längere Zeit mit alkoholischer Kalilauge ohne zu erwärmen stehen, so wird derselbe verseift unter Rückbildung von Camphocarbonsäure. Eine Reduction der Camphocarbonsäure oder ihres Aethers mit Natrium in alkoholischer Lösung analng derjenigen des Camphers zu Borneol ist mir noch nicht gelungen.

Versetzt man die Lösung des camphocarbonsauren Natriums mit Natriumacetat und salzsaurem Phenylhydrazin, so beginnt sehr bald die Ausscheidung einer in schönen Nadeln krystallisirenden Hydrazinverbindung, welche sich jedoch an der Luft leicht verändert, sie wird harzig und färbt sich braun.

Nach den vorhandenen Beobachtungen darf man es als festgestellt anseben, dass der $\mathrm{Camphocarbonsäure} \mathrm{die} \mathrm{Formel} \mathrm{C}_{11} \mathrm{H}_{16} \mathrm{O}_{3}$ zukommt, dass sie ein Carboxyl enthält und dass sie eine Ketonsãure ist.

Die Spaltung von Ketonsäuren beim Erwärmen in wässriger Lösung, wie sie bei der Camphocarbonsäure stattfindet, ist bis jetzt nur bei $\beta$-Ketonsäuren beobachtet; die $\alpha$-Ketonsäuren sind weit beståndiger, Phenylglyoxylsäure zerfällt nur bei der Destillation in Koblensäure und Benzaldehyd. Das Verhalten dieser Säure kann jedoch als Analngiefall zu dem der Camphocarbonsäure angeführt werden und die Beziehung der letzteren als $\alpha$-Ketonsäure zum Campher würde in folgenden Formeln ihren Ausdruck finden:

$$
\begin{array}{ll}
\mathrm{C}_{9} \mathrm{H}_{15} . \mathrm{COH} & \mathrm{C}_{9} \mathrm{H}_{15} \mathrm{CO} . \mathrm{COOH} \\
\text { Campher } & \text { Camphocarbonsäure. }
\end{array}
$$

Der Campher selbst würde denu als Aldehyd zu betrachten sein, für welche Auffassung die Entstehung der Campholensäure (des Oxycamphers) sowobl aus dem Camphoroxim nach Goldschmidt und $Z$ ürrer ${ }^{1}$ ) als auch aus dem $\beta$-Bibromcamplier ${ }^{2}$ ) spricht.

1) Goldschmidt u. Zürrer, diese Berichte XVII, 2069.

2) Kachler u. Spitzer, Monatsh. f. Chem. 188\%, III., 205. 
Der aus Natriumcampher gewonnene Cyancampher Haller's ') ist als das Nitril der Camphocarbonsäure aufzufassen und die aus jenem durch Einwirkung von Kalilauge entstehende Oxycamphocarbonsäure scheint zu der Camphocarbonsäure in derselben Beziehung zu stehen wie die Campholsäure zu Campher:
$\mathrm{C}_{10} \mathrm{H}_{15} \mathrm{O}$. $\mathrm{COOH}$
$\mathrm{C}_{10} \mathrm{H}_{15} \mathrm{O}$. C N
$\mathrm{C}_{10} \mathrm{H}_{17} \mathrm{O}_{2}$. $\mathrm{COOH}$
Camphocarbonsăure
Cyancampher
Oxycamphocarbonsãure

$\begin{array}{lc}\mathrm{C}_{10} \mathrm{H}_{16} \mathrm{O} & \mathrm{C}_{10} \mathrm{H}_{18} \mathrm{O}_{2} \\ \text { Campher } & \text { Campholsăure. }\end{array}$

607. W. Roser: Ueber Phtalylderivate. V. ${ }^{2}$ )

[Mittheilung aus dem chemischen Institut zu Marburg.]

(Eingegangen am 26. November.)

In diesen Berichten theilte ich früher mit, dass bei der Condensation der Bernsteinsäure mit $\mathrm{Phtalsäureanhydrid} \mathrm{ausser} \mathrm{dem} \mathrm{von}$ Gabriel untersuchten Aethindiphtalyl $\mathrm{C}_{18} \mathrm{H}_{10} \mathrm{O}_{4}$<smiles>CCCC1OOC1=CC=C1CC=CO1</smiles>

eine Verbindung $\mathrm{C}_{11} \mathrm{H}_{8} \mathrm{O}_{4}$, das Doppellacton der $\beta$-Benzoylpropion-o-carbonsäure<smiles>O=C1CCC2(COCO2)OO1</smiles>

sodann das $180 a ̈ t h i n d i p h t a l y l \quad \mathrm{C}_{18} \mathrm{H}_{10} \mathrm{O}_{4}$ und eine vierte Verbindung entsteht, welche bei der Trennung der genannten Verbindungen, durch Krystallisation aus Anilin, in Form einer Anilinverbindung ich bezeichne sie als Diphtalsuccinanilid - erhalten wird. In dem Nachstehenden stelle ich die im Verlaufe der Untersuchung jener Verbindungen noch gemachten Beobachtungen zusammen.

1. Derivate des Aethindiphtalyls.

In Alkalien löst sich das Aethindiphtalyl beim Kochen leicht auf, wenn man etwas Alkohol zusetzt; die durch Salzsäure ausgefällte

1) Haller, Jahresbericht 1878, 614.

2) Frühere Mittheilungen verg]. diese Berichte XVII, 2619, 2623, 2770, 2775: diese Berichte XVIII, S02. 